\title{
FENÓMENOS Y PERCEPCIONES ACERCA DE LA PANDEMIA: UN ESTUDIO CON ESTUDIANTES UNIVERSITARIOS EN BRASIL
}

\section{PHENOMENA AND PERCEPTIONS ABOUT THE PANDEMIC: A STUDY WITH UNIVERSITY STUDENTS IN BRAZIL}

\author{
José Lucas MARQUES DUARTE ${ }^{1}$ \\ Paulo FOSSATTI ${ }^{2}$ \\ Hildegard Susana $\mathrm{JUNG}^{3}$ \\ Joanah DAL MAS DOS SANTOS ${ }^{4}$
}

\begin{tabular}{|lll|}
\hline Recibido & $:$ & 14.10 .2021 \\
Aprobado & $:$ & 27.12 .2021 \\
Publicado & $:$ & 31.12 .2021 \\
\hline
\end{tabular}

\begin{abstract}
Resumen: El estudio aborda las percepciones de estudiantes universitarios brasileños sobre la pandemia COVID-19. El objetivo es investigar los efectos de la pandemia en los procesos de enseñanza-aprendizaje y en la salud mental de estudiantes de psicología y pedagogía de una Universidad del sur de Brasil. La metodología es cualitativa, en un estudio de caso con un cuestionario aplicado a estudiantes universitarios. Resultados: de los 57 estudiantes que respondieron, el 75,4\% eran del curso de psicología y el 24,6\% de pedagogía. Cuando se le preguntó cuánto afecta la pandemia al proceso de enseñanza-aprendizaje en la universidad, el 54,38\% respondió que interfiere mucho, el 21,05\% interfiere muchísimo y el 12,5\% interfiere un poco. Se justifican, principalmente, por las respuestas: falta de concentración, falta de intercambio presencial en clase, sobrecarga de actividades en clases online. Para la pregunta relacionada con la salud mental, cuánto afecta la pandemia al desempeño de los estudiantes en el aula, el 50\% respondió que interfiere mucho, el 28,33\% interfiere muchísimo y el 19,33\% interfiere muy poco. Finalmente, se señala que el primer paso para cambiar y transformar los efectos colaterales de la pandemia en la vida de estudiantes universitarios radica en pensar sobre los fenómenos producidos, para abordarlos.
\end{abstract}

Palabras clave: Brasil; Estudiantes Universitarios; Enseñanza-aprendizaje; Salud Mental; Covid-19.

\begin{abstract}
The study addresses the perceptions of Brazilian university students about the COVID-19 pandemic. The aim is to investigate the effects of the pandemic on the teaching-learning processes and on the mental health of psychology and pedagogy students of a University in southern Brazil. The methodology is qualitative, in a case study with a questionnaire applied to university students. Results: from the 57 students who responded, $75.4 \%$ were part of the psychology course and $24.6 \%$ of pedagogy course. When they were asked how much the pandemic affects the teaching-learning process in the university, the $54.38 \%$ answered that it interferes a lot, the $21.05 \%$ that it interferes more than a lot, and the $12.5 \%$ that it interferes very little. They are supported mainly, by the answers: lack of concentration, lack of face-to-face exchange in class, overload of activities in online classes. For the question related to mental health about how much the pandemic affects the performance of students in the classroom, the $50 \%$ answered that it interferes a lot, the $28.33 \%$ that it interferes more than a lot, and the $19.33 \%$ that it interferes very little. Finally, it is pointed out that the first step in order to change and transform the collateral effects of the pandemic in the university students lives, resides in thinking about the phenomena produced, to elaborate them.
\end{abstract}

Key words: Brazil; University Students; Teaching-learning; Mental health; Covid-19.

\footnotetext{
${ }^{1} 1$ Psicólogo. Mestrando em Educação - Universidade La Salle. ORCID: https://orcid.org/0000-0003-4367-0736. E-mail: joselucas_91@yahoo.com.br

2 Psicólogo. Doutor em Educação - Universidade La Salle. ORCID: https://orcid.org/0000-0002-9767-5674. E-mail: paulo.fossatti@unilasalle.edu.br

3 Pedagoga. Doutora em Educação - Universidade La Salle. ORCID: https://orcid.org/0000-0001-5871-3060. E-mail: hildegard.jung@unilasalle.edu.br

4 Graduanda em Psicologia - Universidade La Salle. ORCID: https://orcid.org/0000-0001-7304-8804. E-mail: joanah.dalmas@hotmail.com
} 


\section{INTRODUCCIÓN}

El tema del estudio aborda las percepciones de estudiantes universitarios brasileños sobre la pandemia COVID-19 y se justifica al traer reflexiones sobre este fenómeno histórico-social. Así, el objetivo es investigar los efectos de la pandemia en los procesos de enseñanzaaprendizaje y en la salud mental de estudiantes de psicología y pedagogía de una Universidad del Sur de Brasil.

La educación en Brasil está regulada por la Ley de Directrices y Bases de la Educación Nacional (LDBEN), Ley N. 9.394 (Brasil, 1996), que informa que la educación comprende numerosos procesos de formación, teniendo su desarrollo en la familia, la convivencia humana, el trabajo, instituciones educativas, movimientos sociales y otras organizaciones (Brasil, 1996). Más aún, cuando la LDBEN describe los principios y propósitos de la educación nacional, muestra que la educación es continua y tiene como propósito el desarrollo del estudiante y su calificación, como orienta el artículo 3. "garantía del derecho y al aprendizaje a lo largo de la vida". Asimismo, - "estimular el conocimiento de los problemas del mundo actual, en particular los nacionales y regionales, brindar servicios especializados a la comunidad y establecer una relación recíproca con ella”. Por lo tanto, se investigan los fenómenos involucrados en la salud mental de los estudiantes de una institución comunitaria de educación superior en la región metropolitana de Porto Alegre en medio a la pandemia del COVID-19².

En ese contexto, es saludable pensar en los procesos involucrados en la salud mental de los estudiantes universitarios, así como en las políticas de salud pública, que buscan orientar las formas de acceso y garantía, a través de lineamientos, así como comprender el rol de las universidades para apoyar y ayudar a este universo de estudiantes y, finalmente, cómo la gestión universitaria trabaja con esta temática. En lo que respecta a la salud mental, según la Organización Mundial de la Salud (OMS) el concepto de salud es más que la ausencia de enfermedad, pues solo es posible estar sano cuando existe un completo bienestar físico, mental y social (OMS, 2016). Asimismo, la Organización describe algunos factores que pueden poner en riesgo la salud mental, como cambios sociales, condiciones de trabajo estresantes, discriminación de género, exclusión social, estilos de vida poco saludables, violencia y violaciones de derechos humanos.".

${ }^{2}$ COVID significa Enfermedad del Coronavirus, mientras que "19" se refiere a 2019. Fuente: https://portal.fiocruz.br/pergunta/por-que-doenca-causada-pelo-novo-virus-recebeu-o-nome-de-covid-19. 
En relación a las políticas de salud pública, los involucrados en estos procesos pretenden comprender los aspectos sociales de los individuos, en busca de estrategias que tengan como objetivo transformar la realidad de vida de los sujetos que se encuentran en sufrimiento. Este es un escenario en el que trabaja la psicología, que busca brindar apoyo emocional a los estudiantes, así como ayudarlos a replantear sus ansiedades y/o sufrimientos. Asimismo, la universidad juega un papel fundamental en la construcción de políticas públicas de salud, ya que es un espacio que posibilita discusiones críticas y reflexivas sobre este tema, formando profesionales que conocen sus derechos y pueden acceder, reclamar y orientar a otras personas. Por tanto, la universidad cumple su rol social con los estudiantes y también brinde responsabilidad social con la comunidad, en base al desempeño de estos estudiantes y posteriores profesionales.

En cuanto a la gestión universitaria, se entiende que, debido a los cambios sociales, estas instituciones también experimentan cambios, ya que buscan reinventarse para comprender las necesidades sociales y atender nuevas demandas. Por ello, muchas instituciones emprendedoras contemplan nuevos medios de producir conocimiento, construyendo junto a sus alumnos nuevas formas de enseñar y de aprender. Por ello, es de destacar que las Instituciones de Educación Superior (IES) se sumergen en el mundo tecnológico para contar con subsidios y continuar en el proceso de educación.

Las universidades en general, con ánimo de prevención, buscaron adaptarse al aislamiento social, optando por la enseñanza a distancia, a través de clases sincrónicas con videollamadas. Para estos fines, se han utilizado herramientas y artefactos como: Skype, Google Meet (hangouts), Zoom entre otras plataformas. Se comprende estos recursos como medios preconizados en la LDBEN donde se establece que el poder público incentivará el desarrollo y entrega de programas de educación a distancia, en todos los niveles y modalidades de educación y de educación continua, según la disposición del artículo 80 (Brasil, 1996). Sin embargo, fue necesaria la elaboración de reglamentos complementarios para establecer directrices a la enseñanza remota. Para Alves (2020), El Ministerio de Educación viene publicando ordenanzas desde el 18 de marzo, las cuales se han actualizado constantemente para regular las actividades de los escenarios escolares de educación básica y superior, como las ordenanzas 343, 345, 356 y 473 (Brasil, 2020), suspendiendo las clases presenciales e 
indicando educación remota en caso de emergencia. Por ello, a partir de las implicaciones relacionadas con el COVID-19 y los nuevos modos de organización social, a efectos de combatir la pandemia, se busca relacionar aspectos emocionales de los estudiantes de educación superior con el proceso de enseñanza y aprendizaje en la modalidad online.

\section{Marco Teórico}

La Organización Mundial de la Salud (2016) configura la salud como la plenitud del bienestar, ya sea físico, mental o social. Aquí se cree que es posible divergir de esta conceptualización: el contexto de salud impregna esta completitud, el ser humano es real y su salud depende de numerosos factores, como discuten Gaino et al. (2018), con relación a lo irreal inalcanzable: "[...] Se propone un significado irreal, en el que las limitaciones humanas y ambientales harían imposible alcanzar la condición de "bienestar completo". (p. 110)

Así como el concepto de salud; el concepto de estado de salud mental es empleable en foco de discusiones. Aun así, como explican los autores, sigue prevaleciendo el hecho de que la salud mental es la ausencia de trastornos mentales, ya que estos serían incapacitantes para el individuo, provocando que no lleve una vida sana y de baja calidad. En este sentido, luego de la Reforma Psiquiátrica Brasileña, la problematización de la ausencia total de locura y diagnósticos de trastornos mentales y salud mental se ha problematizado cada vez más. Se corrobora con Gaino et al. (2018) cuando aclaran que: “[...] En este paradigma, la locura es mucho más que un diagnóstico psiquiátrico, ya que los pacientes con un trastorno psiquiátrico pueden tener calidad de vida, participar en la comunidad, trabajar y desarrollar su potencial" (p. 110). Es decir, es importante no naturalizar el estigma de la locura en la sociedad y mirarlo desde cerca para que no suceda.

Por tanto, el contexto de la salud mental impregna el ámbito de la conceptualización, haciéndose presente en las relaciones y en la vida cotidiana de los seres humanos. De esta manera, la problematización y una mirada atenta a la salud mental se perciben no sólo en la comunidad, sino también dentro de la academia. En esta perspectiva, se está de acuerdo con las inferencias de Gaino et al. (2018):

[...] Hablar sobre salud mental en los tiempos actuales significa abordar un área compleja y extensa que va más allá del tratamiento de personas diagnosticadas con 
trastornos mentales. Además, el término salud mental se refiere a los campos del conocimiento, el trabajo técnico y las políticas de salud pública [...]. (p. 115)

Ante esto, la problematización de la salud mental en las IES es importante, sin embargo, se percibe que la discusión va mucho más allá, pues el sistema de este conocimiento es vasto, debiendo ser englobado en su totalidad.

El reconocimiento de la psicología como ciencia fue un proceso que duró años (Zurba, 2011). En esta perspectiva, luego de unos años y su consolidación como área científica, ingresa al campo de la prestación de servicios, surgida desde la función pública y la demanda de la época. En este contexto y tiempo, predominó la aplicabilidad, fundamento básico del funcionalismo, que tuvo un rol orientado al capitalismo, en el que la psicología y toda su funcionalidad se entendía como un producto de mercado, es decir, no se tenía en cuenta las políticas públicas (Zurba, 2011). Poco a poco esta ciencia conquistó espacios de las más diversas formas, haciéndose presente en las siguientes áreas: educativa, social, jurídica, deportiva, hospitalaria, entre otras. De esta manera, en paralelo a estos logros, la mirada crítica de esta profesión también se manifiesta en relación a las políticas públicas que promueven la salud, la singularidad y la dignidad de la persona humana.

En este sentido, tras la pandemia COVID-19 a nivel mundial, la psicología, junto con las ciencias de la salud, jugó un papel fundamental como contingente de las demandas derivadas de este fenómeno. De esta manera, en Brasil, el Consejo Federal de Psicología y los consejos regionales, permitieron flexibilidad en el registro de psicólogos en el sitio web de E-psi, con el fin de aprobar más profesionales en el uso de la atención psicológica en la modalidad online. La nueva pandemia de coronavirus trajo patologías y afecciones específicas que esta enfermedad provoca en los humanos, pero, además, como destacan Silva et al. (2020), la salud mental de los sujetos puede verse afectada de diferentes formas. De esta manera, los impactos psicopatológicos y las enfermedades mentales colectivas se pueden ver en la población brasileña y mundial. Entre los principales hallazgos de Silva et al. (2020), se señala: Asociación de miedo, muerte y dolor con la pandemia; empeoramiento psíquico en el marco de sujetos con condiciones psicopatológicas preexistentes por cuarentena y distanciamiento social; vulnerabilidad psicosocial; pánico general y aumento del estrés, ansiedad y depresión.

Ante lo señalado, se puede evidenciar la importancia de problematizar la enfermedad colectiva que padecía y padece gran parte de la población mundial. Además, es importante que las 
diferentes áreas (especialmente la educación) tomen en cuenta las distintas perspectivas de los grupos sociales, ya que las personas en situaciones de vulnerabilidad tienen escasez de recursos socioeconómicos y de salud (Silva et al., 2020). De esta forma, las universidades que incluyen el curso de psicología han movilizado recepciones en línea, de acuerdo con los lineamientos del código deontológico profesional, con el propósito de ayudar a quienes necesitan asistencia psicológica, tanto docentes, como estudiantes y la comunidad.

Ante estas y otras demandas, es importante pensar en cómo la psicología puede estar cada vez más presente en los diferentes estratos sociales, considerando que la constitución federal, en materia de salud, aboga por el acceso universal para todos. Por tanto, es fundamental desarrollar políticas públicas que se dirijan a estas demandas emergentes.

En el contexto de las políticas públicas, las acciones de las universidades fueron y son fundamentales, ya que permiten reflexiones y discusiones críticas para estas construcciones. Zurba (2011) aclara que, durante la trayectoria de la psicología y el funcionalismo en Brasil, las IES jugaron un papel fundamental en el desarrollo de la ciencia psicológica. Para el autor, fue a partir de este proceso de desarrollo que la universidad impulsó estrategias de intervención en el área de la psicología, en las que lograron sobrevivir a todo este recorrido. De esta forma, como destaca Zurba (2011), las universidades se ocupan de formar al profesional en psicología y no del producto, ya que se produciría "conocimiento único consumible en cada época, y nunca hubiéramos avanzado para repensar nuestros viejos modelos de medidas, intervenciones grupales, formatos de psicoterapia, trabajo de co-terapia, etc.” (Zurba, 2011, p. 27).

Frutoso y Saur (2011) reiteran que las políticas de salud pública estaban enfocadas en el trabajador, es decir, en verificar si estaba apto para el cargo y si no tenía ninguna enfermedad que pudiera perjudicarlo. Se advierte que, en este contexto, la salud brasileña no era accesible para todos. Después de la VIII Conferencia Nacional de Salud, el Movimiento de Reforma en Salud y la Constitución de la República Federativa de Brasil en 1988 (Frutoso y Saur, 2011) se reconfiguró el sistema de salud y el Sistema Único de Salud (SUS) se convirtió en una clave de acceso de toda la población brasileña a salud. Actualmente, el SUS es ampliamente estudiado y discutido en la comunidad y también en la academia. Dicho esto, es fundamental discutir la trayectoria de cada carrera sobre las perspectivas de las políticas públicas y el SUS dentro de las universidades. 
Paulo et al. (2018) enfatizan la relación que el desarrollo comunitario y la globalización impactan en la educación. Para los autores, es relevante llevar la discusión de la gestión universitaria al contexto académico institucional y organizacional, ya que las instituciones de educación superior no solo son impulsoras de la docencia, de la investigación y de la extensión, sino también del capital humano. Así como la sociedad, cambian la educación superior y las Instituciones de Educación Superior (IES), como lo exponen Dewes y Bolzan (2018). Este cambio interno que aportan los autores refleja la comprensión práctica y subjetiva del funcionamiento de las IES. Corrobora el concepto de gestión universitaria derivado de Dewes y Bolzan (2018) cuando se refieren a la gestión de los tiempos y de los espacios formativos, es decir, contemplando no sólo el contexto educativo, sino todo el proceso que abarca el ámbito de la educación superior, incluidos los administrativos y burocráticos, ya que en este contexto se desarrollan las acciones del docente universitario y de los estudiantes. Comprender la funcionalidad de las IES y la gestión universitaria es relevante para mejor comprender la gama de voces de los agentes incluidos en este escenario.

Machado y Freitas (2018) consideran el diálogo como una herramienta importante para la construcción de la enseñanza y del aprendizaje. En este sentido, la pregunta es: ¿la tecnología y la situación de cuarentena en la pandemia interfieren en el diálogo en el aula en línea? Las autoras reiteran que a través de la gestión del educador se erige la responsabilidad de ayudar a construir una conciencia crítica de sí mismo y del mundo (Machado y Freitas, 2018). Por tanto, en este momento de crisis no solo en salud, sino en economía y educación, en el que el mundo atraviesa varias transformaciones, sobre todo subjetivas, el educador asume el papel de cuestionador: noticias, información y conocimiento científico teórico.

Desde esta perspectiva, Ribeiro et al., (2013) corroboran cuando exponen la importancia del proceso educativo dejando la posición de colonialidad, en la que aún persisten en la actualidad. Los autores enfatizan que, al igual que la educación para la salud, la transformación es lenta, pero avanza hacia una construcción efectiva, especialmente a través de las relaciones. Por tanto, para ellos, el proceso educativo se desarrolla "en las relaciones entre las personas que enseñan y que aprenden, en las que se evita caer en una relación jerárquica de superioridad e inferioridad en ellos" (Ribeiro et al., 2013, p. 168). 


\section{Metodología}

Esta investigación es de naturaleza cualitativa y su tipología corresponde a un estudio de caso, con un cuestionario aplicado a estudiantes universitarios de cursos de psicología y pedagogía en una Universidad del Sur de Brasil. Así, el cuestionario fue aplicado online por Google Forms.

Según Gil (2008), las investigaciones caracterizadas como estudios de caso están relacionadas con técnicas cualitativas en el análisis del material. Para el autor, el análisis cualitativo presenta tres etapas: reducción de los datos, presentación y conclusión/verificación. El paso de reducción ejemplifica que debe haber un proceso de selección para que se pueda reducir los datos a analizar, por lo tanto, es algo que ocurre no solo en la selección de las respuestas al cuestionario, por ejemplo, sino también en la selección de datos teóricos del material de construcción de este artículo. La presentación corresponde a la organización de los datos seleccionados, colaborando en el análisis de datos. Se puede realizar de diferentes formas y utiliza diferentes recursos, como gráficos, tablas, cuadros, textos, entre otros. El último paso es la conclusión/verificación, que requiere una revisión detallada de todo el proceso de construcción de la obra, con el fin de verificar que todo el análisis de los datos obtenidos está cubierto a lo largo del artículo.

El estudio de caso, según los autores Lüdke y André (1986) tiene como objetivo descubrir, interpretar el contexto, retratar la realidad de manera completa y profunda, además de representar puntos de vista conflictivos presentes en una situación social. Corroborando lo anterior, Will (2012) destaca que el estudio de caso puede favorecer la comprensión de un fenómeno o situación más amplia, como es el objetivo de este estudio. Así, Gil (2008) también se refiere al estudio de caso como una investigación empírica que investiga un fenómeno actual dentro de su real contexto, cuando los límites entre el fenómeno y el contexto no están claramente definidos y en el que se utilizan múltiples fuentes de la evidencia.

Fueron elegidos como sujetos participantes en la encuesta los estudiantes de las carreras de psicología y pedagogía debido a la facilidad de accesibilidad de los autores del presente artículo. Así, el cuestionario, armado utilizando el recurso Google Forms, contiene 5 preguntas, siendo 3 de ellas cerradas y 2 abiertas. Fue enviado por medio del WhatsApp (de modo privado y grupos) el 29 de marzo de 2021 y el 12 de abril de 2021. El formulario se mantuvo abierto, 
aceptando respuestas desde el 29 de marzo de 2021 hasta el 14 de abril de 2021. Obteniendo 57 respuestas en total.

\section{Tabla 1}

Preguntas enviadas a los estudiantes universitarios

\begin{tabular}{l|l}
\hline Curso & Pedagogía o Psicología \\
\hline $\begin{array}{l}\text { ¿Cuánto afecta la pandemia al proceso de } \\
\text { enseñanza-aprendizaje en la universidad? }\end{array}$ & $\begin{array}{l}\text { Nada interfiere; Poco interfiere; Indiferente; } \\
\text { Interfiere mucho o Interfiere muchísimo }\end{array}$ \\
\hline Justifique su respuesta & Respuesta abierta \\
\hline $\begin{array}{l}\text { En cuanto a la salud mental, ¿cuánto } \\
\text { afecta la pandemia al rendimiento de los } \\
\text { estudiantes en el aula? }\end{array}$ & $\begin{array}{l}\text { Nada interfiere; Poco interfiere; Indiferente; } \\
\text { Interfiere mucho o Interfiere muchísimo }\end{array}$ \\
\hline Justifique su respuesta & Respuesta abierta \\
\hline
\end{tabular}

Fuente: elaboración propia (2021).

A la luz de lo anterior, hay que destacar que las preguntas están ordenadas según la Escala Likert de 1 a 5 , así: nada interfiere 1 , interfiere levemente 2 , indiferente 3 , interfiere mucho 4 e interfiere muchísimo 5. A continuación se presentan los resultados y discusiones de los datos obtenidos del cuestionario con estudiantes universitarios.

\section{Resultados y discusión de los datos}

Con la pandemia de coronavirus hubo cambios en el mundo y, en consecuencia, en la educación superior. Una palabra en este caótico momento estuvo más presente que nunca en la vida de los estudiantes: adaptación. Esta adaptación se refiere a las actividades y clases presenciales que migraron a la pantalla de un teléfono celular y/o una computadora. Según Brito y Fofonca (2019), las tecnologías digitales de la información y la comunicación colaboran para importantes transformaciones en la educación, ya que permiten la construcción de nuevos escenarios y fomento de un cambio de paradigma.

Por tanto, a partir de este apartado, se discuten los resultados obtenidos con estudiantes de una institución de educación superior en el sur de Brasil, en la que se problematiza, a través de la triangulación (datos obtenidos, inferencias y lo que dicen los autores del marco teórico) todas estas transformaciones y adaptaciones necesarias en este período de educación a distancia. 
Como resultado de la aplicación del cuestionario online se obtuvieron 57 respuestas, siendo la mayoría de los participantes estudiantes del curso de Psicología $(75,4 \%)$ y el $24,6 \%$ del curso de Pedagogía.

A continuación, se presenta la pregunta que contempla la percepción de los estudiantes sobre el proceso de enseñanza-aprendizaje y la pandemia: “¿Cuánto afecta la pandemia al proceso de enseñanza-aprendizaje en la Universidad?’. Los resultados muestran que para el 54,38\% de los estudiantes la pandemia interfiere mucho en el proceso de enseñanza-aprendizaje en la universidad, y para el $21,05 \%$ de los estudiantes interfiere muchísimo. Por otro lado, las respuestas muestran que poco interfiere para el $14.03 \%$, indiferente el $8.77 \%$ y nada interfiere el $1,75 \%$.

Según Nhantumbo (2020), no es sencillo para el estudiante la adopción de tecnologías de la información, pues él se prepara para clases presenciales en las que recibe los materiales en un ambiente donde hay contacto directo con el docente en el aula. En este sentido, es evidente que existen implicaciones para la relación profesor-alumno en el ambiente remoto, ya que las interacciones se restringen a la relación online, lo que también implica el proceso de enseñanzaaprendizaje. Es de destacar que el rol del docente va más allá de solo tener habilidades técnicas, ya que la relación humana también engloba interacciones que atraviesan lo subjetivo de los sujetos en las relaciones interpersonales.

Las justificaciones a las respuestas a la pregunta se clasificaron en dos bloques, como muestra la Tabla 2 a continuación.

\section{Tabla 2}

Respuestas a la cuestión discursiva con respecto a la pandemia y el proceso de enseñanza y aprendizaje

\begin{tabular}{|c|c|}
\hline Categoría I - Agotamiento & Categoría II - Sociabilidad \\
\hline $\begin{array}{l}\text { E8 } 8^{3} \text { - "Estaba acostumbrada y me resultó } \\
\text { muy fácil aprender, compartir y participar en } \\
\text { clase con la enseñanza presencial. Con el } \\
\text { inicio de la pandemia y la enseñanza } \\
\text { migrando a remoto, se vuelve agotador estar }\end{array}$ & $\begin{array}{l}\text { E7 - "Falta contacto con compañeros y } \\
\text { profesores y convivencia con lo que la } \\
\text { universidad puede ofrecer. Pero el contacto } \\
\text { online hace posible el aprendizaje, hay } \\
\text { conferencias. Y el contacto online con el }\end{array}$ \\
\hline
\end{tabular}

\footnotetext{
${ }^{3}$ Los participantes han sido numerados aleatoriamente, de forma secuencial, y se les ha atribuido la letra "E", de "estudiante". Las respuestas han sido traducidas al español. 
frente a la pantalla ... Interesante informar que noté que la participación de compañeros en clase disminuyó en un 80\%”.

E10 - "Clases con mayor número de alumnos, retraso en el inicio de clases, exceso de actividades que realizar y poca actividad en el aula, como explicación del contenido de parte de los profesores".

E12 - "La carga mental de permanecer en la habitación todo el día trabajando y luego estudiando sin tener otras ocupaciones y distracciones ha resultado ser muy difícil. Tuve que volver a tomar mi medicación para la ansiedad y me siento muy agotada".

E17 - "Estoy un poco desmotivada porque la situación de la pandemia es mala aquí en Brasil y ya tengo mis problemas personales".

E18 - "El cansancio, la tensión, todo dificulta la concentración en los estudios".

E19 - "Interfiere demasiado. Estoy extremadamente abrumada y, a menudo, me falta el coraje para abrir la computadora y seguir el ritmo de las clases, y mucho menos la avalancha de trabajos y lecturas que se requiere de nosotros, los estudiantes. Es muy difícil, no logro mantenerme saludable”.

E29 - "Enseñar y aprender está muy relacionado con una mente descansada, sana... Y con todo lo que estamos viviendo, a veces se hace muy dificil prestar atención en las clases online y realizar el trabajo con la misma eficacia que antes de la pandemia".

E30 - "Las clases en formato remoto terminan por cansar al alumno y desmotivarlo. No prestamos atención como si estuviéramos en el aula. Una hora de clase termina pareciendo dos. $Y$ algunos profesores acaban sin tomarse un descanso porque están en modo remoto y eso es muy agotador. Si hay pausas en persona, ¿por qué no las hay de forma remota?". grupo, profesor y compañeros, por tanto, interfiere mucho porque hay una falta de mayor contacto, lo que trae mayor interacción en su conjunto".

E11 - "Por la situación en la que nos encontramos. No puede estar del todo bien. Miedo a perder familiares, falta de contacto con los demás. Somos seres humanos complejos, y lo que afecta a una parte, afecta a todo".

E13 - "Pérdida de convivencia y dificultades de concentración y aprendizaje en la docencia a través de pantallas".

E14 - "La educación se mantuvo con calidad, sin embargo, entiendo que en persona el intercambio de conocimientos sería mayor y más significativo".

E20 - "Creo que interfiere la falta de interacción entre colegas y profesores en las aulas. Hay mucho estrés para administrar varios trabajos y más cada día. A menudo ni siquiera tenemos espacio para clases en línea. $Y$ administrar tantos trabajos "compensatorios" no es fácil, porque con la pandemia estamos corriendo aún más para obtener ganancias ya que el país está en crisis".

E23 - "De hecho, podría haber una opción de "interfiere", pero lo más cercano sería "interfiere mucho". Creo que afecta las relaciones personales y los intercambios con compañeros y profesores, las socializaciones $e$ incluso las horas de clase, que online acaban siendo más reducidas, ya que las clases a distancia son realmente agotadoras.".

E25 - "Específicamente con respecto a mi aprendizaje, el contacto humano en el aula es muy necesario".

E26 - "Además de no interactuar con profesores, compañeros, suele haber intervenciones en internet". 
E48 - "El aumento de la demanda de actividades online desborda las actividades académicas que ya se realizaban a través de tecnologías digitales".

E51 - "Manejo bien el modo en línea, pero es más difícil mantener la concentración, los maestros están ofreciendo más actividades y las pasantías son mucho más limitadas".
E31 - "El estado emocional de las personas está bastante afectado ... sin mencionar la nueva rutina de home office".

E32 - "El cambio de cara a cara a online, la falta de contacto visual, todo dificulta, porque a través del vídeo solo vemos la cara. No podemos tener una visión más amplia ... La calidad de la enseñanza se ve comprometida".

E35 - "Creo que en los intercambios con profesores en clases presenciales es mucho más productivo".

La tabla muestra las justificaciones para marcar la respuesta "¿Cuánto afecta la pandemia al proceso de enseñanza-aprendizaje en la universidad?".

Fuente: Elaboración propia en base a respuestas al cuestionario (2021).

Las respuestas en la categoría I muestran que los estudiantes están más cansados que en las clases presenciales, además de estar sobrecargados con la forma como se estuvo llevando a cabo la educación en la modalidad remota. Por tanto, podemos reflexionar que muchos docentes no estaban preparados para enseñar en línea, resultando en el desarrollo de muchas actividades como vía compensatoria del proceso de enseñanza-aprendizaje.

Otro aspecto para destacar, que proviene del aislamiento social, así como de la pandemia, es la dificultad entre la separación del espacio de estudio/trabajo con el espacio de hogar/descanso/ocio. En este sentido, podemos reflexionar que antes de que se estableciera la pandemia, existían separaciones de los innumerables espacios y actividades, pero con la pandemia se unificaron todos los espacios. En otras palabras, se puede decir que ya no existe una separación física y, por tanto, tampoco se percibe una separación subjetiva, lo que puede llevar al malestar emocional, puesto que los procesos ya no pueden percibirse como cíclicos y finitos.

Corroborando lo expuesto y en línea con los datos de los estudiantes, Morales y López (2020) señalan la somatización del reflejo pandémico y todo el contexto que ya vivía el alumno, incluida la incertidumbre sobre el regreso a clases, la inseguridad que acecha en el caso del retorno, y la carga de trabajo asociada al tiempo de encierro. Así, el impacto de esta situación pandémica puede causar daños no solo en un aspecto de la vida de este estudiante, sino en 
varios, como enfatizan los autores. Además, en los resultados obtenidos en su estudio, Morales y López (2020) definen cinco categorías en las que los estudiantes demostraron un impacto significativo de la pandemia en su rutina, a saber: malestar psicológico, rendimiento académico, acceso a clases en línea, adquisición de contenidos e interacción. con los docentes, lo que refuerza no sólo los hallazgos de la categoría uno, que se aporta en este trabajo, sino también en las demás, que se discuten a continuación.

En la categoría II, en función de las respuestas de los estudiantes, existen implicaciones para los intercambios personales, las relaciones interpersonales y la interacción de los estudiantes con sus compañeros y con los profesores. Se observa que los encuestados afirman que el proceso de enseñanza-aprendizaje sufre interferencias, pues entienden que no se encuentran bien emocionalmente. Por tanto, se cita Brait et al. (2010):

Las relaciones humanas, aunque complejas, son elementos fundamentales en el desempeño profesional y conductual de un individuo. Así, el análisis de las relaciones entre profesor / alumno involucra intenciones e intereses, y esta interacción es el eje de las consecuencias, ya que la educación es una de las fuentes más importantes del desarrollo conductual y un elemento agregador de valores en los miembros de la especie humana. (p. 2)

En consecuencia, se argumenta que las relaciones humanas promueven vínculos de transferencia que posibilitan el vínculo entre el deseo de enseñar y aprender del docente y del alumno. Por tanto, los roles que se asumen en estas relaciones no son solo de reproducción técnica, sino también de intercambios subjetivos, resignificaciones y elaboraciones psíquicas.

En este contexto, Neves y Lemos (2019) señalan que, incluso en la modalidad a distancia, es necesario que las relaciones no se deben distanciar: "La educación a distancia se basa en procedimientos que permiten el establecimiento de un proceso de enseñanza y aprendizaje incluso cuando no existe un contacto cara a cara entre los sujetos" (p. 336). Los autores también reiteran que basarse en modelos y principios de acción es fundamental para establecer un entorno seguro, cercano y de apoyo para estos estudiantes. Para ello, parten de la teoría de los afectos del filósofo Spinoza, ya que se correlacionan emociones y afectos, y la voluntad no tiene libre albedrío, ya que existe dependencia entre sujetos y afectos y todo esto sucede porque el hombre se ve afectado por el mundo que lo rodea (Neves y Lemos, 2019). Por tanto, esta afectación en el ámbito universitario se da en el aula, en las relaciones con los compañeros, con 
los profesores, con las reflexiones e intercambios que se hacen con todos y las innumerables otras formas en que los estudiantes pueden incidir dentro del contexto académico.

Se destaca que estas interferencias se dan no solo en la diferenciación de relaciones, frente al ambiente del aula, sino también en el proceso de enseñanza-aprendizaje, especialmente en lo que se refiere a la salud mental y asistencia emocional de estos estudiantes. Por lo tanto, la tabla 3 indica en sus resultados la intensidad con la que la pandemia COVID-19 afecta el desempeño de los estudiantes en el aula. Según Schimidt et al. (2020):

[...] Las incertidumbres sobre cómo controlar la enfermedad y sobre la gravedad del COVID-19, además de la imprevisibilidad sobre la duración de la pandemia y sus consecuencias, se caracterizan como factores de riesgo para la salud mental de la población en general. (p. 7)

A partir de esto, se puede ver la influencia de la pandemia en la salud mental de la población, así como en la salud mental de los estudiantes.

Continuando con los resultados y sus discusiones, en la pregunta (tabla 3 referente a la salud mental y si la pandemia afectó de alguna manera el desempeño en clase, el $50 \%$ de los estudiantes respondió que interfiere mucho y el 28,33\% que interfiere muchísimo. El 19,33\% dijo que interfiere poco, el 3,33\% de los estudiantes dijo que es indiferente. Ninguno de los estudiantes marcó "nada interfiere".

Una encuesta realizada por Maia y Dias (2020) con estudiantes portugueses demuestra que la pandemia afectó la salud mental de estos sujetos, señalando niveles significativos de estrés, ansiedad y depresión en el período pandémico, en comparación con el período normal (2018 y 2019). Como se sabe, el impacto en la salud mental puede provocar una caída en el rendimiento en clase y, como señalan los autores, estos efectos pueden o no ocurrir durante un largo período de tiempo. Por ello, Maia y Dias (2020) también reiteran la importancia de los estudios y prácticas de prevención y/o remediación para ayudar a estos universitarios, lo que corrobora lo discutido en el marco teórico y los resultados obtenidos con los encuestados.

Por lo tanto, con respecto a la recopilación de datos sobre salud mental, se preguntó a los estudiantes lo siguiente: "En cuanto a la salud mental, ¿cuánto afecta la pandemia al rendimiento de los estudiantes en clase?". 


\section{Tabla 3}

Respuestas a la cuestión discursiva sobre salud mental durante la pandemia y el desempeño del estudiante

Categoría III - Salud mental, síntomas y
trastornos

E9 - "Creo que debido a la preocupación de que todos sean sometidos al aislamiento social, debe haber una ansiedad mínima".

E13 - "El aislamiento y la reclusión favorecen el desarrollo de trastornos como la depresión, el TDAH, entre otros".

E16 - "Estoy muy ansiosa, comiendo más y extraño a amigos y familiares, me siento más triste".

E17 - "Estoy respondiendo basándome en cómo me siento. Pero con respecto a otros colegas, observo que hay mucha interferencia de la pandemia en la salud mental".

E20 - "No hay forma de dedicarte como debería. Mi salud mental está en la unidad de tratamiento intensivo".

E22 - "Se producen sentimientos de gran tristeza ... desesperanza".

E26 - "Altos índices de ansiedad".

E32 - "Nerviosismo, desánimo y apatía".

E36 - "Siento que he estado muy ansiosa, molesta e irritada últimamente. En conversaciones con otros colegas, noto que el sentimiento es compartido por otros. El hecho de que no tengamos una perspectiva concreta de "volver a la normalidad" en un
E4- "El hecho de que estemos en una situación delicada, donde necesitas estar en casa y cuando salgo a trabajar si tienes miedo, acaba generando diferentes problemas psicológicos, depende de quién sea. Pero el caso es que todo esto es nuevo, por lo que nadie está preparado para afrontar la situación, en consecuencia, la adversidad ocurre y puede desencadenar problemas en la psique. Esto crea problemas en todas las áreas de la vida, especialmente en el aprendizaje.".

E5- "Es visible que hay un impacto, más por la presión de no contraer el virus que por estudiar a distancia. Sucede que ambos se mezclan".

E11- "Los estudiantes están experimentando una pandemia, con muchas personas muriendo, un momento de lamentaciones. Están viviendo solos, no pueden salir a ver a sus amigos. El rendimiento en clase disminuye porque no ven una dirección $n i$ motivos para estudiar".

E14- "La educación a distancia nos da una mayor libertad en relación a las clases y actividades, por eso, la falta de rutina, personalmente, termina convirtiéndose en un factor para que ocurra la desorganización y la culpa por no poder manejar todo y termina siendo un sentimiento imperante".

E15- "La pandemia interfiere en diversos ámbitos de nuestra vida, la salud mental es un ámbito de gran impacto, dado que estamos en alerta constante ante una enfermedad que es nueva en nuestra sociedad. Otro factor que incide en la salud mental es la mala gestión de los políticos durante este período de crisis, ya que son 
momento dado comienza a pesarnos y las personas se sienten cansadas a este ritmo".

E37 - "Sí, afecta mucho, porque si el alumno no puede contar con el apoyo de un profesional de la salud (para someterse a terapia), durante sus dificultades emocionales, ya que estamos viviendo una época difícil y dependiendo de la estructura familiar, es muy complicado".

E38 - "Creo que la pandemia ha interferido enormemente con la ansiedad y la salud mental de los estudiantes, ya que incluso en las clases algunos compañeros citan el daño causado durante este momento difícil. El momento actual ha perjudicado a todos, a veces es complicado estudiar con familiares enfermos, además de una excesiva preocupación, que hace que los estudiantes no puedan obtener el $100 \%$ de su concentración".

E39 - "Debido al aislamiento, al no tener contacto con compañeros, amigos y familiares, esto puede desencadenar depresión, ansiedad que también puede agravarse por el aislamiento social y la preocupación por contagiarse el virus y transmitirlo a la familia”.

E49 - "Las dificultades que eran comunes al entorno académico pueden reforzarse $y$ fortalecerse. Con la sobrecarga emocional se desarrollan diferentes dificultades como ansiedad, insomnio, depresión etc.".

52 - "Todos están sobrecargados, además de la necesaria dedicación a la universidad, necesitan lidiar con sobrecarga laboral, duelo, conflictos internos y con conocidos, problemas familiares, violencia e incluso todo el contexto político. Es difícil tener mucha energía para los estudios".

53 - "Creo que todos estamos cansados de las circunstancias en las que vivimos, nos enfrentamos a diversas pérdidas (familia, trabajo, estabilidad económica) y sin duda figuras que deben apoyar a la ciudadanía en materia de salud, ingresos, vivienda, educación y acceso a la información".

E18- "Creo que las clases online para muchos son menos efectivas, no es lo mismo aprender así que aprender en persona. Además del hecho de que probablemente ya esté cansado de esta situación pandémica, es algo que desgasta a todos e incluso deja a algunos sin perspectivas de un futuro mejor".

E23 - "¡Por la pérdida de seres queridos!”

E24 - "iSe justifica simplemente por el hecho de que estamos en una pandemia! En su mayor parte, vivimos al borde de problemas que nos desgastan mentalmente.".

E27 - "Además de la dificultad económica, algunos estudiantes y familiares perdieron su fuente de ingresos o hubo una reducción muy alta de ella. Otros miembros de la familia están trabajando en casa, los que tienen hijos tienen que estar cerca, esto dificulta a los que están estudiando y otras actividades que causan estrés".

E29 - "La incertidumbre del futuro y el miedo a la situación actual impiden que el alumno se concentre bien, además de imposibilitar que nos dediquemos tanto como antes".

E30 - "La pandemia trajo varios desafios, como problemas financieros y psicológicos (emocionales) y el tema de los plazos y la cantidad de trabajo no se está mitigando (en algunas asignaturas). Creo que todos estamos pasando por algo en este momento, y sería interesante pensar "con más cariño" sobre eso. No solo para los estudiantes, sino también para los profesores".

E35 - "Creo que estoy expuesto a un virus que no se conoce, lo que te puede pasar y a los miembros de tu familia". 
todas ellas tienen un impacto significativo en la salud mental de los estudiantes y reflexionan sobre su desempeño en las asignaturas".
E40 - "También hay mucho cariño. Además de cambiar todos los métodos de enseñanza, nuestra psicología afecta con noticias, miedos y ansiedades".

E44 - "Ante la realidad de que cada día vamos perdiendo gente a nuestro alrededor, es complicado tener que seguir con tanto dolor. También sabiendo que muchos están contrayendo el virus y teniendo secuelas. Al reflexionar sobre cómo será el día siguiente y quién será el próximo en ser golpeado, incluso uno mismo".

E45 - "La pandemia genera miedo, depresión y ansiedad, factores que influyen directamente en el desempeño de los estudiantes en el aula".

E46 - "Estrés por estar en casa, acumulación de tareas en casa, trabajo y universidad, miedo por haber perdido a miembros de la familia".

E47 - "La falta de contacto con otras personas, la incertidumbre de los eventos y la propia pandemia pueden haber obstaculizado el desempeño, desencadenando ansiedad, depresión y los trastornos asociados a este momento".

La tabla muestra las justificaciones para marcar la respuesta "Con respecto a la salud mental, ¿cuánto afecta la pandemia al desempeño de los estudiantes en clase?".

Fuente: Elaboración propia en base a respuestas al cuestionario (2021).

Las respuestas de las Categorías III y IV indican una preocupación significativa de los estudiantes por el aislamiento social y la pandemia de COVID-19, además de las dificultades para mantener el aprendizaje en modo remoto. Se perciben implicaciones en relación al aumento de la "ansiedad” y “estrés", así como la manifestación de otros síntomas, favoreciendo el desarrollo de enfermedad mental.

Otro aspecto presente en las respuestas es la referencia al término angustia, dado que puede entenderse como la manifestación del sufrimiento psíquico. Porque cuando no se vive lo que le genera satisfacción, y/o sentido, la persona entra en ese estado de sufrimiento, pudiendo incluso desarrollar un cuadro clínico de enfermedad. En otras palabras, la angustia ocupa un 
lugar de sufrimiento, cuando no es posible vivir lo que uno quiere. En este sentido, el aislamiento social y los miedos a la enfermedad física y/o mental se vinculan a la pandemia y a las percepciones de la vida ante las nuevas demandas, de manera que cada sujeto afronte sus males individuales y psicosociales, en la forma que considere la mejor, y no siempre con la ayuda de un profesional de la psicología.

A partir de esta comprensión, se piensa en la producción y experimentación del duelo, como lo demuestran las respuestas de los estudiantes. El duelo se expresa como pérdidas y finitudes, tales como: a) trabajos; b) financiero; c) contacto social; d) la relación con el otro; e) la muerte de los seres queridos. Según Meireles (2016 apud Bowlby, 1990) el duelo se caracteriza por cuatro fases: 1) Entumecimiento; 2) Anhelo; 3) Desorganización; 4) Reorganización. Así, el duelo es el proceso inevitable de elaboración de una pérdida, que despierta una amplia gama de sentimientos y cambios que invaden e interfieren con el funcionamiento emocional de una persona. En conclusión, la pandemia se considera un proceso de duelo, vivido por todos, ya que la angustia y los miedos que provoca traspasan las barreras físicas y llegan a todos los estratos sociales. Por tanto, se afirma que existen impactos en la salud mental de los estudiantes de las IES, que van más allá de las adaptaciones técnicas y tecnológicas.

\section{CONCLUSIONES}

A la vista de los resultados presentados por los estudiantes y las reflexiones derivadas de las lecturas, se notó que la pandemia tuvo efectos significativos en la vida de estos estudiantes, especialmente en lo que se refiere al proceso de enseñanza-aprendizaje, la salud mental y su desempeño en clase.

La afectación entre personas, aunque sea de forma virtual, es diferente del compartir cara a cara con los compañeros. Otros temas como el agotamiento, el cambio de atención y pensamiento, acumulación de tareas y muchas veces falta de materiales para acompañar las clases estuvieron presentes en la pregunta sobre el proceso de enseñanza-aprendizaje en el período pandémico. Con respecto al desempeño en clase y la salud mental, las preocupaciones sobre la salud y la contaminación, el duelo, el desempleo y la situación política en Brasil fueron comunes en las respuestas. Además, en esta pregunta, palabras como ansiedad, apatía, depresión, nerviosismo, cansancio, tristeza, desesperanza, estrés y tensión se destacaron en las respuestas de los estudiantes. También se pudo notar que se repiten muchas respuestas a las preguntas abiertas, 
lo que puede interpretarse como un vínculo entre los efectos de la pandemia en los procesos de enseñanza-aprendizaje, la salud mental y el desempeño de los estudiantes.

Si bien las consecuencias de la pandemia en la vida académica están presentes y pueden durar mucho tiempo, problematizar y ser consciente de este impacto es importante para que las acciones de intervención, promoción y prevención se puedan pensar junto con la academia. Finalmente, se señala que el primer paso para cambiar y transformar los efectos colaterales de la pandemia en la vida de los estudiantes universitarios radica en pensar sobre los fenómenos producidos, para elaborarlos.

Como limitación de este artículo se indica la gran cantidad de respuestas de los estudiantes, lo que demuestra la necesidad de que se expresen sobre la experiencia vivida en el ámbito académico y el impacto de la pandemia en el mismo. Como aporte e indicaciones de futuro, el artículo enfatiza la importancia de prestar mucha atención a las preguntas sobre el proceso de enseñanza-aprendizaje y la salud mental de los estudiantes, por lo que aún es necesario monitorear a estos estudiantes. A diferencia del año 2020 y el inicio de la pandemia, la academia tendrá nuevos desafíos, como si antes la inserción de estas tecnologías y nuevos medios de aprendizaje dentro de los hogares de los estudiantes, ahora el desafío será insertarse nuevamente en el aula, lo que provoca no solo ansiedad, miedo, entre otros, sino también una nueva mirada a este alumno que nuevamente tendrá que adaptarse, es decir, ¿cómo se verá impactada la relación entre la universidad y los estudiantes?

\section{REFERENCIAS}

Alves, L. (2020). Educação remota: entre a ilusão e a realidade. Interfaces CientíficasEducação, 8(3), 348-365. https://periodicos.set.edu.br/educacao/article/view/9251

Brait, L. F. R., de Macedo, K. M. F., da Silva, F. B., Silva, M. R. y de Souza, A. L. R. (2010). A relação professor/aluno no processo de ensino e aprendizagem. Itinerarius Reflectionis, 6(1), 1-15. https://www.revistas.ufg.br/rir/article/view/40868

Brasil. (1996). Lei $n^{\circ}$ 9.394, de 20 de dezembro de 1996. Estabelece as Diretrizes e Bases da Educação Nacional. http://www.planalto.gov.br/ccivil_03/leis/19394.htm

Brito, G. da S. y Fofonca, E. (2019). Educação a distância e seus paradigmas numa sociedade em trânsito: em busca de uma identidade metodológica. In. Santos, S. C. A. dos, Cavalcante, I. F., Lemos, E. das C., Ferreira, M. da C. y Costa, M. L. da (Orgs.). Educação e Sociedade: formação profissional, educação a distância e tecnologias (pp. 367-387). 1. ed. São Luís, MA: Editora IFMA. 
Dewes, A. y Bolzan, D. P. V. (2018). Gestão universitária a partir da narrativa de professores gestores de departamentos didáticos. Regae: Revista de Gestão e Avaliação Educacional, 7(15), 39-53. https://www.redalyc.org/journal/4718/471857005004/html/

Frutoso, J. T. y Saur, B. (2011). O Sistema Único de Saúde brasileiro e a clínica ampliada. In. Zurba, M. do C. (Org.). Psicologia e saúde coletiva (pp. 41-50). Florianópolis: Tribo da Ilha.

Gaino, L. V., Souza, J. de, Cirineu, C. T. y Tulimosky, T. D. (2018). O conceito de saúde mental para profissionais de saúde: um estudo transversal e qualitativo. Revista Eletrônica Saúde Mental Álcool e Drogas, 14(2), $108-116$. https://www.revistas.usp.br/smad/article/view/149449

Gil, A. C. (2008). Métodos e técnicas de pesquisa social. 6. ed. São Paulo: Atlas.

Lüdke, M. y André, M. (1986). Pesquisa em educação: abordagens qualitativas. Em Aberto, 5(31), 17-24. http://emaberto.inep.gov.br/ojs3/index.php/emaberto/article/view/1971

Machado, M. E. y Freitas, A. L. S. de. (2018). Diálogos em roda: contribuições para a formação de educadores e educandos na educação formal e não formal. Revista Educação Por Escrito, $9(1)$ 54-65. https://revistaseletronicas.pucrs.br/ojs/index.php/porescrito/article/view/28056

Maia, B. R. y Dias, P. C. (2020). Ansiedade, depressão e estresse em estudantes universitários: o impacto da COVID-19. Estudos de Psicologia, 37, e200067, 1-8. https://www.scielo.br/j/estpsi/a/k9KTBz398jqfvDLby3QjTHJ/?lang=pt

Meireles, I. O. (2016). O Luto na Fase Adulta: Um Estudo Sobre a Relação Apego e Perda na Teoria de John Bowlby. Revista Ciências Humanas, 9(1), ed. 16, 92-105. https://doi.org/10.32813/2179-1120.2016.v9.n1.a274

Morales, V. J. y Lopez, Y. A. F. (2020). Impactos da pandemia na vida académica dos estudantes universitários. Revista Angolana de Extensão Universitária, 2(3), esp., 53-67. https://www.portalpensador.com/index.php/RAEU-BENGO/article/view/205

Neves, T. T. y Lemos, E. das C. (2019) Afetando a educação: primeiros passos. In. Santos, S. C. A. dos, Cavalcante, I. F., Lemos, E. das C., Ferreira, M. da C., Costa, M. L. da (Orgs.). Educação e Sociedade: formação profissional, educação a distância e tecnologias (pp. 331366). 1. ed. Editora IFMA.

Nhantumbo, T. L. (2020). Capacidade de resposta das instituições educacionais no processo de ensino-aprendizagem face à pandemia de Covid-19: impasses e desafios. EducamazôniaEducação, Sociedade e Meio Ambiente, 25(2), 556-571. https://periodicos.ufam.edu.br/index.php/educamazonia/article/view/7851

Organização Mundial de Saúde (OMS). (2016). Saúde mental depende de bem-estar físico e social, diz OMS em dia mundial. https://brasil.un.org/

Paulo, M. C. C. de., Costa, D. de M., y Andrade, R. M. M. de. (2018). Desafios da gestão universitária contemporânea: presenteísmo e seus impactos no desenvolvimento discente. 
$\begin{array}{llll}\text { Revista } & \text { GUAL, } & \text { 11(4), } & \text { ed. }\end{array}$ https://www.redalyc.org/articulo.oa?id=319357027001

Ribeiro, D., Souza, E. S., Sousa. F. R. de, Teixeira, I. M. de C. y Oliveira, M. W. de. (2013). Educar-se com grupos, organizações e movimentos sociais: processos educativos em práticas sociais populares. Revista Pedagógica, 15(31), 165-181. https://doi.org/10.22196/rp.v15i31.2335

Silva, H. G. N., Santos, L. E. S. dos y Oliveira, A. K. S. de. (2020). Efeitos da pandemia do novo Coronavírus na saúde mental de indivíduos e coletividades. Journal Of Nursing And Health, $10(4)$ $1-10$. https://periodicos.ufpel.edu.br/ojs2/index.php/enfermagem/article/view/18677/11415

Will, D. E. M. (2012). Metodologia da pesquisa científica: livro digital. 2. ed. rev. e atual. UnisulVirtual.

Zurba, M. do C. (2011). Trajetórias Da Psicologia Nas Políticas Públicas De Saúde. In. Zurba, M. do C. (Org.). Psicologia e saúde coletiva (pp. 25-37). Florianópolis: Tribo da Ilha. https://psicologia.paginas.ufsc.br/files/2012/06/Miolo_Psicologia-e-Saude.pdf 